\title{
Dual Targeting of Cell Growth and Phagocytosis by Erianin for Human Colorectal Cancer
}

This article was published in the following Dove Press journal:

Drug Design, Development and Therapy

\author{
Yihan Sun (D) \\ Guofeng Li $\mathbb{D}^{\prime}$ \\ Qi Zhou (D)' \\ Danyue Shao (1D) ${ }^{2}$ \\ Jingwei $\mathrm{Lv}^{3}$ \\ Jianhua Zhou (D)
}

'School of Traditional Chinese Medicine, Changchun University of Chinese Medicine, Changchun I30II7, People's Republic of China; ${ }^{2}$ Second School of Clinical Medicine, Beijing University of Chinese Medicine, Beijing 100029, People's Republic of China; ${ }^{3}$ jilin Ginseng Academy, Changchun University of Chinese Medicine, Changchun I30II7, People's Republic of China
Correspondence: Jianhua Zhou School of Traditional Chinese Medicine, Changchun University of Chinese Medicine, 1035 Boshuo Road, Jingyue National High-Tech Industrial Development Zone, Changchun I30II7, People's Republic of China Tel +86-15948000805

Email jianhuazhoul334@sohu.com
Objective: To investigate the effect of erianin on tumor growth and immune response in human colorectal cancer cells (CRC).

Methods: The effect of erianin on tumor growth was determined by CCK8 and colony formation assay. Western blotting was used to evaluate the expression levels of relevant proteins and qRT-PCR was used to evaluate the mRNA level of the relevant gene. The transcriptional activity of $\beta$-catenin was determined by dual-luciferase reporter assay. Cellular thermal shift assay was used to quantify drug-target interactions. The cell surface CD47 was assessed by flow cytometry. The enrichment of H3K27 acetyl marks on CD47 promoter was evaluated by chromatin immunoprecipitation assay. Phagocytosis assay was used to determine the phagocytic activity of macrophage. In vivo role of erianin was studied on xenograft models.

Results: We found that erianin significantly decreased cell survival, colony formation, induced cell cycle arrest, and led to cell apoptosis in SW480 and HCT116 cells. Mechanism analysis demonstrated that erianin inhibited the nuclear translocation and transcriptional activity of $\beta$-catenin, which might result from erianin- $\beta$-catenin interaction. In addition, the downstream gene expressions, such as c-Myc and cyclin D1, was decreased. More interestingly, erianin decreased the expression of CD47 by regulating H3K27 acetyl marks enrichment on CD47 promoter. Consequently, macrophage-mediated phagocytosis was increased. Our in vivo experiments further confirmed the inhibitory effect of erianin on tumor growth.

Conclusion: In summary, erianin could inhibit CRC cells growth and promoted phagocytosis, which suggested erianin as a potential therapeutic strategy for CRC patients.

Keywords: erianin, colorectal cancer, $\beta$-catenin, CD47, macrophage

\section{Introduction}

Colorectal cancer (CRC) remains the third most prevalent cancer type and leading cause of cancer-related deaths with 1.8 million cases and 862,000 deaths worldwide during 2018. ${ }^{1}$ The occurrence and progression of CRC result from a wide array of cellular transformation processes, which include genetic and epigenetic mutations that drive uncontrolled cell proliferation and escape from apoptosis. ${ }^{2-4}$ Chemotherapy and surgery remain the major therapeutic treatment for $\mathrm{CRC}$ patients. ${ }^{5}$ Fluoropyrimidine-based chemotherapy (eg, 5-fluorouracil) has been used as the first-line systemic chemotherapy of treating advanced CRC for over a half century. ${ }^{6}$ However, most patients receiving chemotherapy finally develop drug resistance, which is considered to be the major reason for CRC therapy failure. ${ }^{7}$ Furthermore, even though chemotherapy has significant antitumor activity, the side effects can affect the quality of a patient's life, which makes the new therapeutic approaches urgent. 
Traditional Chinese medicines, such as Dendrobium, have been shown to exert anticancer activity in many kinds of cancers. ${ }^{8,9}$ Erianin (2-methoxy-5-[2-(3,4,5-trimethoxyphe-nyl)-ethyl]-phenol; Figure 1A), a natural compound derived from Dendrobium candidum, shows various pharmacological activities and therapeutic potential to inhibit multiple cancers in vivo and in vitro. ${ }^{10-16} \mathrm{Li}$ et al demonstrated that erianin inhibited the proliferation of acute promyelocytic leukemia HL-60 cells by regulating the expression of bcl-2 and bax. ${ }^{10}$ In addition, erianin caused moderate growth delay in xenografted human hepatoma Bel7402 and melanoma A375. ${ }^{11}$ Furthermore, erianin induced cell cycle G2/M-phase arrest and apoptosis via the JNK signalling pathway in osteosarcoma and bladder cancer. ${ }^{12,13}$ Erianin can also inhibit cell invasion, metastasis and angiogenesis in lung cancer and breast cancer by the
A<smiles>COc1ccc(CCc2cc(OC)c(OC)c(OC)c2)cc1O</smiles>

Erianin, C18H22O5; molecular weight, 318.36.
B

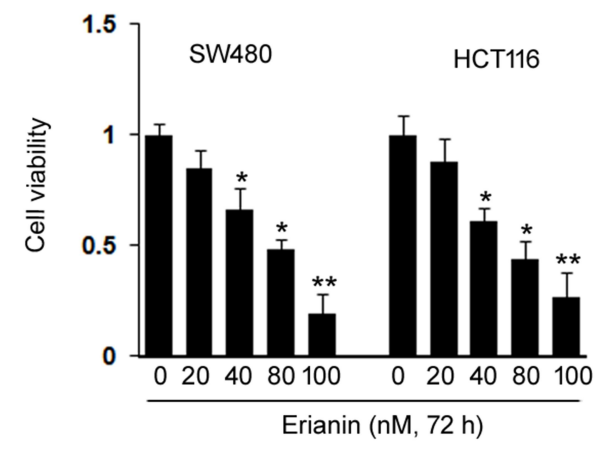

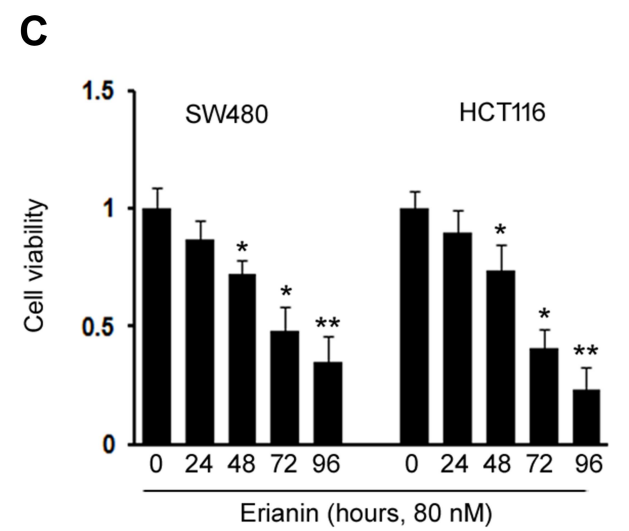

D
$\mathbf{E}$

NCM460

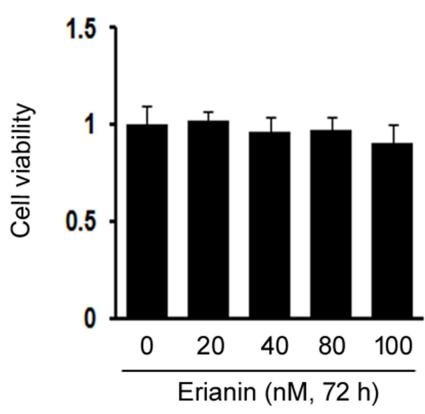

NCM460

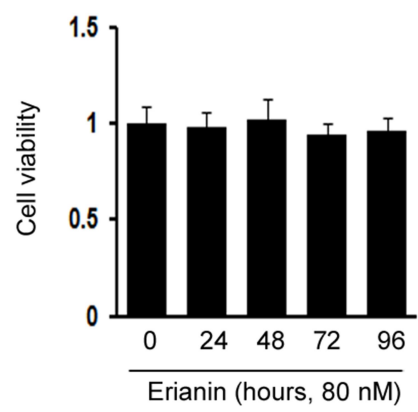

$\mathbf{F}$ 0 20
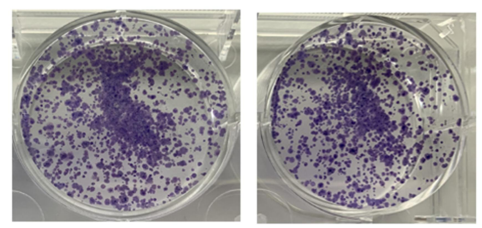

40
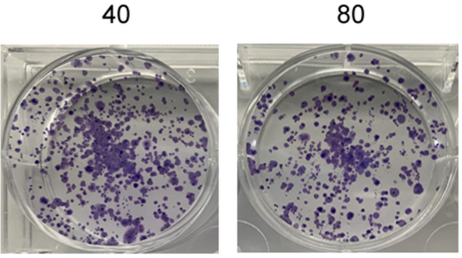

100

Erianin (nM)
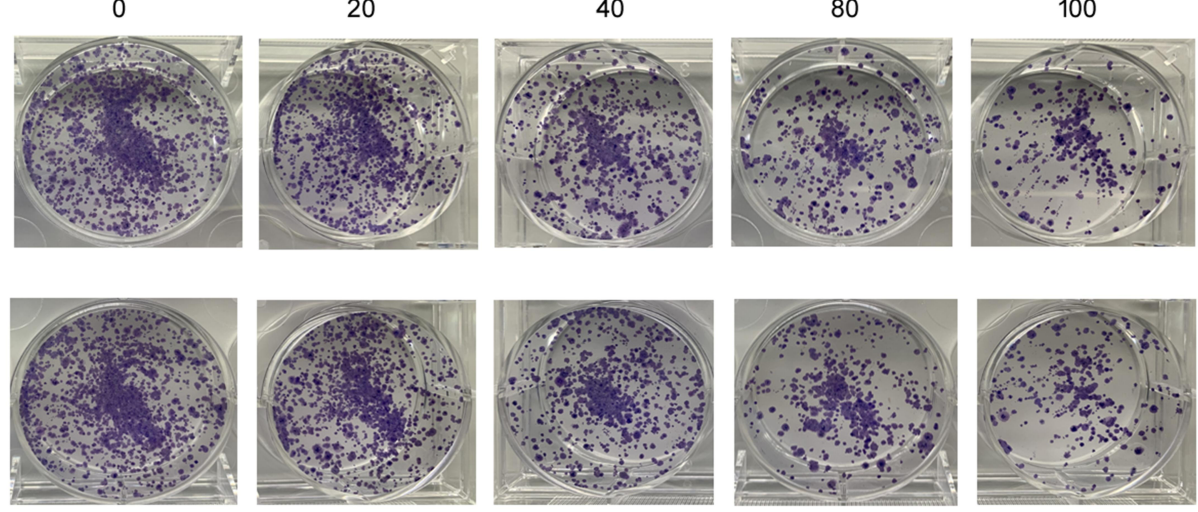

Figure I Erianin inhibited CRC cells growth. (A) Chemical structure of erianin. (B and C) SW480 and HCTII6 cells were treated with indicated concentration (B) and time (C) of erianin, cell viability was assessed by CCK8 assay. ${ }^{* P}<0.05$, ${ }^{*} * P<0.01$. (D and E) NCM460 cells were treated with indicated concentration (D) and time (E) of erianin, cell viability was assessed by CCK8 assay. (F) SW480 and HCTII 6 cells were performed colony formation assay after being treated with indicated concentration of erianin. 
regulation of IDO, MPP and TIMP expressions. ${ }^{14,15}$ Interestingly, besides the function on cell growth, apoptosis, and migration, erianin was found to strongly affect the serum levels of cytokines and immune response in liver cancer. ${ }^{16}$ More importantly, in addition to the anticancer effects, previous a study also suggested that erianin had no major organ-related toxicities. ${ }^{12}$

However, to the best of our knowledge, neither the mechanism nor the effect of erianin on colorectal cancer has been reported. Hence, in this study, we evaluate the antitumor potential and molecular mechanisms of erianin in human colorectal cancer SW480 and HCT116 cells and provide a theoretical basis of erianin application for colorectal cancer therapy.

\section{Materials and Methods Materials}

Antibodies against cleaved PARP (cat \# 5625), Bak (cat \# 12105), Bax (cat \# 14796), Bcl-2 (cat \# 15071), Bcl-xL (cat \# 2764), $\beta$-catenin (cat \# 8480), cyclin D1 (cat \# 55506), c-Myc (cat \# 18583), HDAC2 (cat \# 57156), and GAPDH (cat \# 5174) were purchased from Cell Signaling Technologies (Danvers, MA, USA). Antibody against $\alpha$-tubulin (cat \# T6199) was purchased from Sigma Aldrich Co. (St Louis, MO, USA).

Erianin was purchased from Shanghai Yuanye BioTechnology Co., Ltd (China) and dissolved in DMSO. Wnt/ $\beta$-catenin signaling inhibitor WNT974 was purchased from MedChemExpress (Monmouth Junction, NJ USA) and dissolved in DMSO.

\section{Cell Culture}

The human colorectal cancer cell lines SW480 and HCT116 were purchased from American Type Culture Collection (ATCC, Manassas, VA, USA). Cells were maintained in RPMI1640 medium supplemented with 10\% FBS (Thermo Fisher Scientific, Waltham, MA, USA), $100 \mathrm{U} / \mathrm{mL}$ penicillin and $100 \mu \mathrm{g} / \mathrm{mL}$ streptomycin (Thermo Fisher Scientific) and cells were cultured at $37^{\circ} \mathrm{C}$ with $5 \% \mathrm{CO}_{2}$.

\section{Cell Viability and Colony Formation Assay}

Cell viability was assessed with the Cell Counting Kit 8 (CCK8, Dojindo, Japan) according to the manufactorer's instructions.

For the colony formation assay, CRC cells (1000 cells/ well) were seeded in a six-well plate and maintained in medium for 10-14 days. Subsequently, the colonies were fixed with $4 \%$ paraformaldehyde and stained with $0.1 \%$ crystal violet, and the number of clones was counted using an inverted microscope.

\section{Quantitative Real-Time PCR (qRT-PCR)}

Total RNA from CRC cells was isolated using RNA isolation kit (Omega, Norcross, GA, USA) according to the manufacturer's protocol. Total RNA $(1 \mu \mathrm{g})$ was used as the template for cDNA synthesis by using iScript $^{\mathrm{TM}}$ Reverse Transcription Super mix kit (Bio-Rad Laboratories Inc., Hercules, CA, USA) before the samples were analyzed using SYBR green master mix on a real-time PCR system (Bio-Rad Laboratories Inc.). The primer sequences used were as follows: c-Myc, forward 5'AAACACAAACTTGAACA

GCTAC-3', reverse 5'- ATTTGAGGCAGTTTACATT ATGG-3'; cyclin D1, forward 5'-AGGCGGATGAGAAC AAGCAGA-3', reverse 5'-CAGGCTTGACTCCAGAAG GG-3'; CD47, forward 5'-GGCAATGACGAAGGAGGT TA-3', reverse 5'-ATCCGGTGGTATGGATGAGA-3'; and GAPDH, forward 5'-CACCCACTCCTCCACCTTTG-3' and reverse 5'-CCACCACCCTGTTGCTGTAG-3'. The $2-\Delta \Delta \mathrm{Cq}$ method was used to calculate the relative expression levels.

\section{Western Blotting}

For Western blotting, $20 \mu \mathrm{g}$ cellular protein extracts were separated in SDS-PAGE gel and were then transferred to nitrocellulose membranes (EMD Millipore, Burlington, MA, USA). The membrane was blocked with 5\% non-fat milk and incubated with primary antibodies overnight at $4^{\circ}$. Then, the membranes were incubated with secondary antibody and the proteins were visualized using Super Signal West Pico Chemiluminescent Substrate (Thermo Fisher Scientific).

\section{Transit Transfection}

Plasmid pEGFP-N1-beta-catenin was purchased from Addgene (Watertown, MA, USA). Lipofectamine 2000 (Thermo Fisher Scientific, Carlsbad, CA, USA) was used for transit transfection according to the instructions.

$\beta$-catenin siRNA was purchased from Sigma-Aldrich Co. Lipofectamine RNAiMAX (Thermo Fisher Scientific) was used for transfection according to the instruction. 


\section{Cell Cycle Analysis}

After treated with vehicle or indicated drugs, CRC cells were harvested by trypsinization, fixed with $70 \%$ ethanol, and retained at $-20^{\circ} \mathrm{C}$ overnight. After cells were centrifuged and washed with PBS, they were resuspended in propidium iodide (PI) solution containing RNase $(100 \mu \mathrm{g} / \mathrm{mL})$ in the dark at room temperature for $30 \mathrm{~min}$ and then studied in a flow cytometer.

\section{Caspase-3/7 Activity Assay}

Apo-ONETM homogeneous caspase-3/7 assay (Promega Corporation, Madison, WI, USA) was used to measure caspase-3/7 activity. Briefly, Apo-ONE ${ }^{\circledR}$ homogeneous caspase-3/7 reagent ( $100 \mu \mathrm{L} /$ well) was added to a 96-well plate, and the plate was then placed on a shaker for five minutes (300-500 rpm) before incubating for $12 \mathrm{~h}$ at room temperature. The reading of each well was measured by spectrofluorometer.

\section{Apoptosis Assay by Annexin V}

Annexin V-FITC staining was used to detect the extent of apoptosis induced by erianin. Briefly, CRC cells were treated with erianin for $48 \mathrm{~h}$, and were then collected and resuspended in $200 \mu \mathrm{L}$ annexin V-binding buffer and $5 \mu \mathrm{L}$ PI for 10 minat room temperature in the dark. Then the cells were finally analyzed by the flow cytometry (BD FACS Calibur) with an emission filter of $600 \mathrm{~nm}$ for PI (red) and 515-545 nm for FITC (green).

\section{Apoptosis Assay by DAPI}

The effect of erianin on apoptosis induction was evaluated by DAPI staining assay. CRC cells $2 \times 10^{3}$ were seeded in a 96-well plate. After treatment, the cells were washed three times with PBS and 4\% paraformaldehyde was added to each well for fixation. After permeabilization with TritonX-100 solution $(0.1 \%)$, DAPI solution $(0.1 \%)$ was added. The cells with condensed and fragmented chromatin were analyzed by ECHO fluorescence microscopy.

\section{Cellular Thermal Shift Assay}

For cellular thermal shift assay, CRC cells were pretreated with $10 \mu \mathrm{M}$ MG132 for one hour, and then incubated with erianin for four hours. After washing with ice-cold PBS, cells were aliquot into PCR tubes $(100 \mu \mathrm{L}$ each) and incubated at different temperatures for four minutes. After being frozen and thawed twice using liquid nitrogen, cells were centrifuged and proteins were analyzed by Western blotting.

\section{TOP/FOP Luciferase Reporter Assay}

The transcriptional activity of $\beta$-catenin was assessed using the TOP/FOP dual-luciferase reporter system (DualGlo $^{\text {TM }}$ Luciferase Assay System, Promega). The Renilla luciferase plasmid pRLTK (Promega), which controls for transfection efficiency, was cotransfected with $\beta$-cateninresponsive firefly luciferase reporter plasmid TopFlash (EMD Millipore) or the negative control FopFlash (EMD Millipore) using the lipofectamine 2000 (Thermo Fisher Scientific). Cells were harvested after $24 \mathrm{~h}$ in culture and the luciferase activity was determined by the Luciferase Assay System (Promega) using a Microplate Luminometer (Berthold, Bad Wildbad, Germany).

\section{Flow Cytometry Analysis}

Erianin treated CRC cells were washed, and resuspended in $100 \mu \mathrm{L}$ FACS buffer and stained with FITC-conjugated anti-CD47 (BD Biosciences, San Jose, CA, USA) antibodies. All samples were incubated for 30 minutes at $4{ }^{\circ} \mathrm{C}$, and then washed twice with FACS buffer. Flow cytometry analyses were performed on BD FACS Canto II.

\section{In Vitro Phagocytosis Assay}

For phagocytosis assay, THP1 derived macrophages were seeded in a six-well tissue culture plate. Erianin-treated CRC cells were washed and labeled with $2.5 \mu \mathrm{M}$ of carboxyfluorescein succinimidyl ester (CFSE) (Thermo Fisher Scientific). After incubating macrophages in serumfree medium for two hours, CFSE-labeled CRC cells were added to the macrophages for another two hours at $37^{\circ} \mathrm{C}$; macrophages were then washed and imaged with an inverted microscope. The phagocytosis efficiency was calculated as the number of macrophages containing CFSElabeled CRC cells per 100 macrophages.

\section{Chromatin Immunoprecipitation (ChIP) Assay}

ChIP assays were performed using the SimpleChIP ${ }^{\circledR}$ Enzymatic Chromatin IP Kit (Cell Signaling Technologies) according to manufacturer's instructions using the antibodies against H3K27AC. Immunoprecipitated DNA was analyzed by QRT-PCR using the following primers: CD47 promoter fragment 1: F: 5'-AGGATGAATGATGTGGCCTGT-3' and R: 5'- CAAACAGGCATTAGCAGCGT-3'; fragment 2: F: 
5'-GGGGATGTGTTGGATACGCT-3' and R: 5'- CTCTG CGTTCGGCTCGTCTA-3'; fragment 3: F: 5'-AGGGAAG AGCAGAGCGAGTA-3' and R: 5'- TTGCTTTCACTCC CACCCTC-3'; fragment 4: F: 5'-AGAGAGAGGACAG TGGGGC-3' and R: 5'- CCAGTCGCAGGCTCCAGA-3'; fragment 5: F: 5'- GCCGCGTCAACAGCA-3' and R: 5'AAAGGCATCATTCTTGGAAATTGT-3'.

\section{In Vivo Xenograft}

NOD/SCID (Shanghai Slac Laboratory Animal Co., Ltd, China) mice were injected (subcutaneously in right flank) with $5.0 \times 10^{6}$ SW480 cells per mouse suspended in $75 \mu \mathrm{L}$ PBS and mixed with an equal volume of matrigel. Animals with tumors (volume $\sim 100 \mathrm{~mm}^{3}$ ) were divided into two groups (n=6) and treated with either placebo or $50 \mathrm{mg} / \mathrm{kg}$ erianin for continuously three weeks by intraperitoneal injection. Tumor size were measured at the indicated times. All the animal-related procedures were approved by the Animal Care and Use Committee of The Changchun University of Chinese Medicine. All animal experiments were conducted according to the NIH Guide for the Care and Use of Laboratory Animals.

\section{Statistical Analysis}

Data were presented as mean \pm SD from three independent experiments. $P$ value was determined using paired Student's $t$-test, and a $P$ value $<0.05$ was deemed to indicate statistical significance.

\section{Results}

\section{Erianin Inhibited CRC Cell Growth}

Figure 1A illustrates the chemical structure of erianin. To investigate the inhibitory effect of erianin on CRC cell viability, we treated two CRC cell lines (SW480 and HCT116) with different concentrations of erianin $(0,20,40,80$, and $100 \mathrm{nM})$ for 24, 48, 72, and $96 \mathrm{~h}$. As shown in Figure 1B and C, erianin treatment significantly inhibited the viability of CRC cells in a dose- and time-dependent manner. Importantly, erianin did not show cytotoxic effects on normal human colon mucosal epithelial cell line, NCM460 (Figure 1D and E). In addition, consistent with the short-term growth assay, our colonyforming unit assay also showed that erianin inhibited the colony formation ability of SW480 and HCT116 cells (Figure 1F).

\section{Erianin Elevated Cell Cycle Arrest and Apoptosis}

To verify the causal relation of cell viability inhibition, the cell cycle distribution was analyzed. Erianin increased cell number at $\mathrm{G} 2 / \mathrm{M}$ phase, but decreased cell number at $\mathrm{S}$ and $\mathrm{G} 0 / \mathrm{G} 1$ phases after 24-h incubation with indicated concentration in SW480 and HCT116 cells (Figure 2A and B). To explore the effect of erianin on apoptosis, we examined the activity of caspase 3/7, the protein level of cleaved PARP, Bax, Bak, Bcl2, and Bcl-xL. As shown in Figure 2C-E, the activity of caspase 3/7, protein level of cleaved PARP, Bak, and Bax (proapoptosis) increased as the concentration of erianin increased. In contrast, the protein level of Bcl-2 and Bcl-xL (antiapoptotic) decreased after erianin treatment (Figure 2E). Annexin V flow cytometry and DAPI staining further confirmed that erianin could induce cell apoptosis (Figure 2F and G).

\section{Erianin Inhibited $\beta$-Catenin Translocation} Increasing evidence revealed that the $\mathrm{Wnt} / \beta$-catenin pathway plays critical role in colorectal cancer tumorigenesis. We hypothesized that erianin might have effect in modulating the $\mathrm{Wnt} / \beta$-catenin pathway. First, we investigated the effect of erianin on $\beta$-catenin phosphorylation. As shown in Figure 3A, no obvious change was observed on $\beta$-catenin phosphorylation level. We then evaluated the effect of erianin on $\beta$-catenin translocation. As shown in Figure 3B-E, $\beta$-catenin expression in cytoplasm was increased, whereas expression in the nucleus was decreased with the treatment of erianin in a doseand time-dependent manner. To further explore the effect of erianin on $\beta$-catenin transcription activity, we performed TOP/FOP dual luciferase assay. We found that TOP/FOP relative luciferase activity was significantly decreased after erianin treatment both in SW480 and HCT116 cells (Figure 3F and G).

\section{Erianin Bound $\beta$-Catenin Directly}

Since erianin inhibited $\beta$-catenin translocation to the nuclear without changing its phosphorylation level, we hypothesized that erianin might bind $\beta$-catenin directly. To determine whether erianin physically binds $\beta$-catenin, we performed a cellular thermal shift assay. The results from this experiment indicated that erianin treatment increased the thermal stability of $\beta$-catenin when cells were pretreated with the proteasome inhibitor MG132 for one hour (Figure 4A and B). In contrast, erianin treatment had no effect on the thermal stability of GAPDH, a loading control (Figure 4A and $\mathrm{B})$. These results strongly suggested a specific physical interaction between erianin and $\beta$-catenin. 


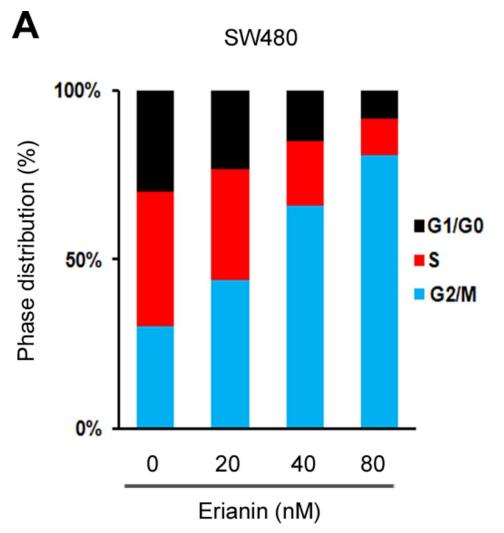

D

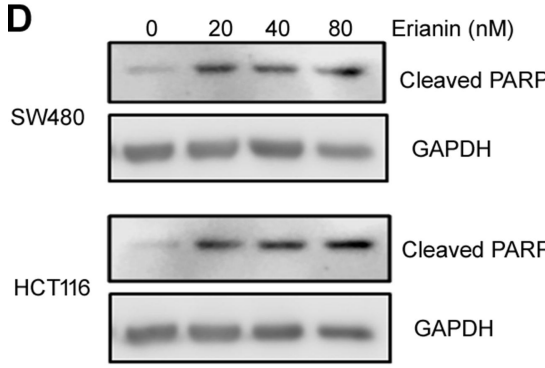

B

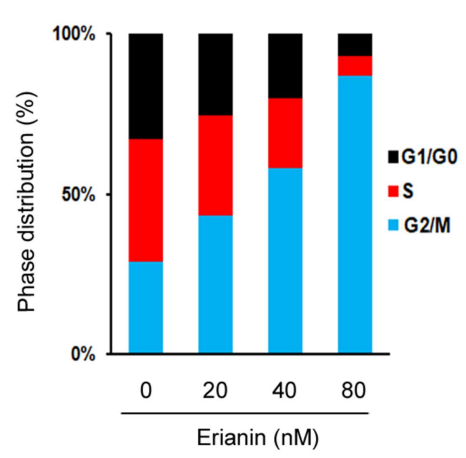

E
C

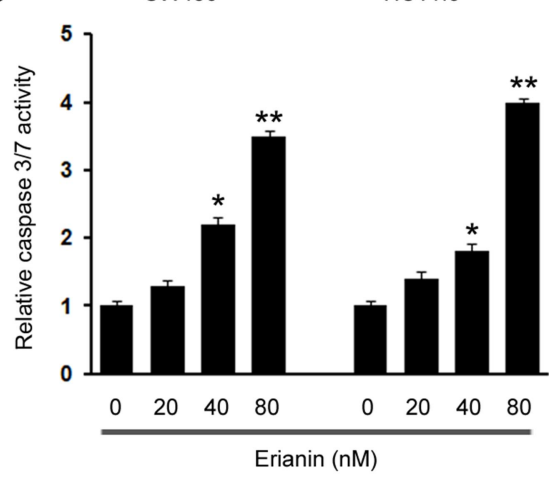

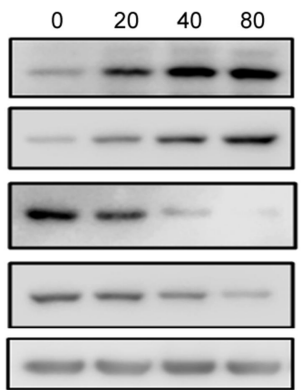
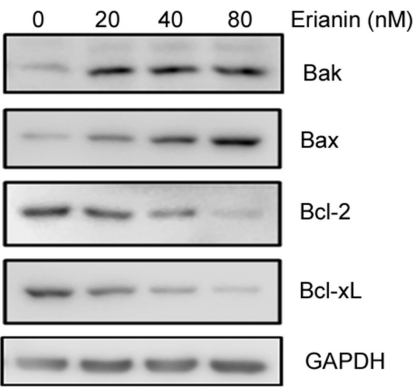

SW480 HCT116

F
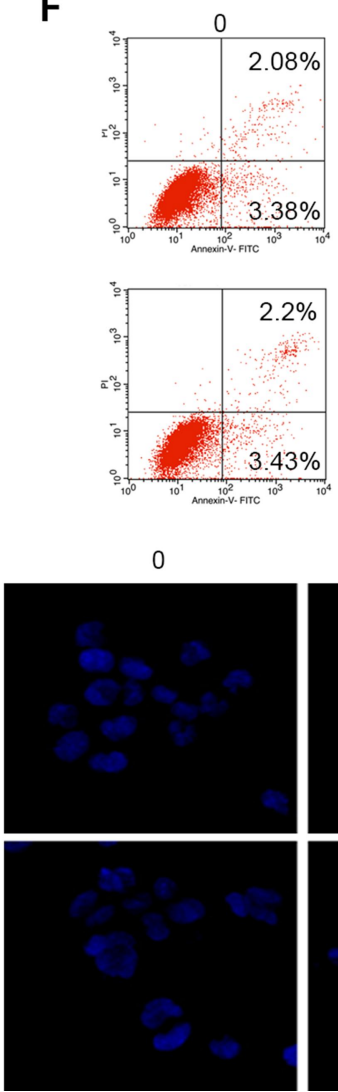
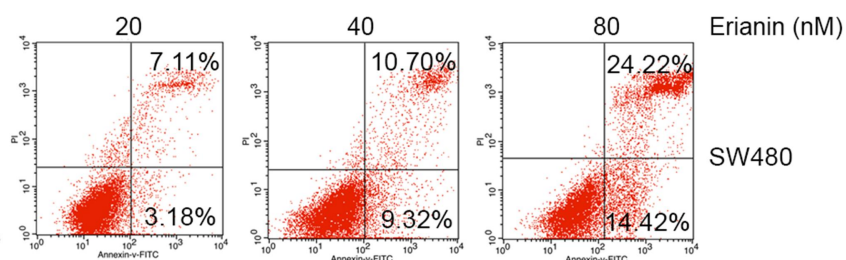

G

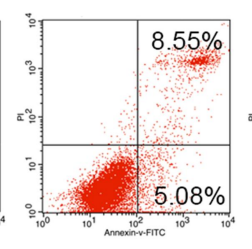

20
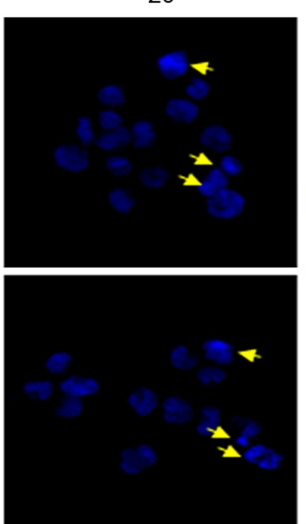
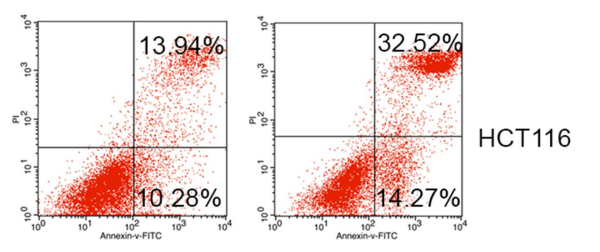

40
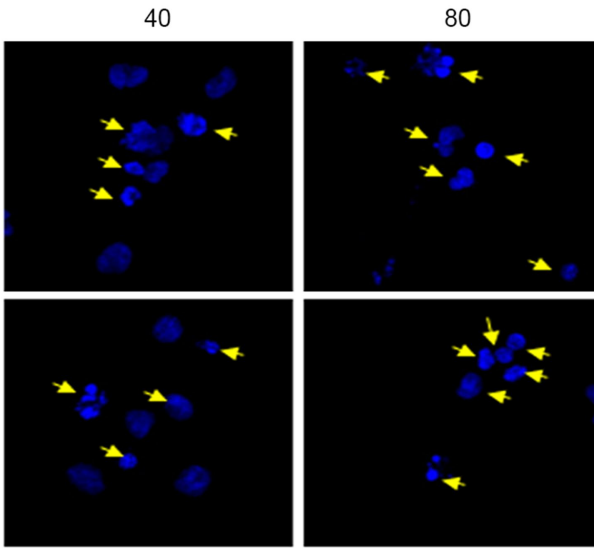

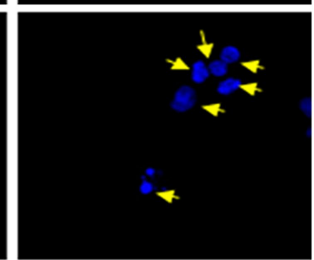

Erianin (nM)

Erianin (nM)

SW480

HCT116

Figure 2 Erianin elevated cell cycle arrest and apoptosis. (A and B) SW480 and HCTII6 cells were treated with erianin for $48 \mathrm{~h}$ and then analyzed by PI staining to determine cell cycle phase distribution. (C) SW480 and HCTII6 cells were treated with erianin for $48 \mathrm{~h}$, the relative caspase-3/7 activity was measured using Apo-One ${ }^{\mathrm{TM}}$ homogenous caspase-3/7 assay. $* P<0.05$, $* * P<0.01$. (D and E) The protein level of cleaved PARPI, Bak, Bax, Bcl-2 and Bcl-xL were analyzed by Western blotting after treated with indicated concentration of erianin. (F and G) SW480 and HCTII6 cells were treated with erianin for 48 h, apoptosis was assessed using annexin-V flow cytometry analysis (F) or DAPI staining (G). 
A

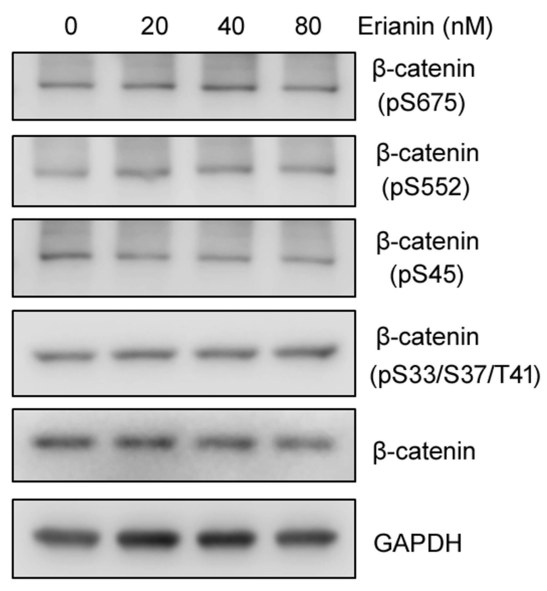

D

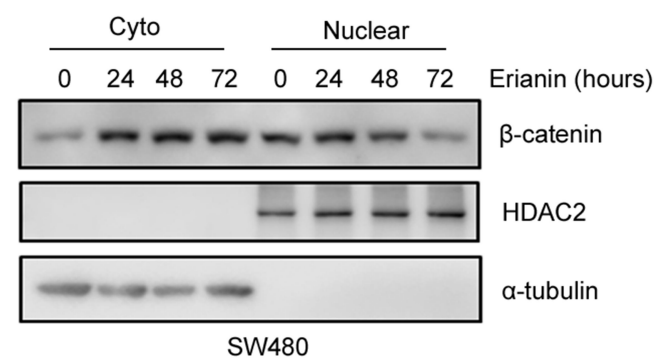

$\mathbf{E}$

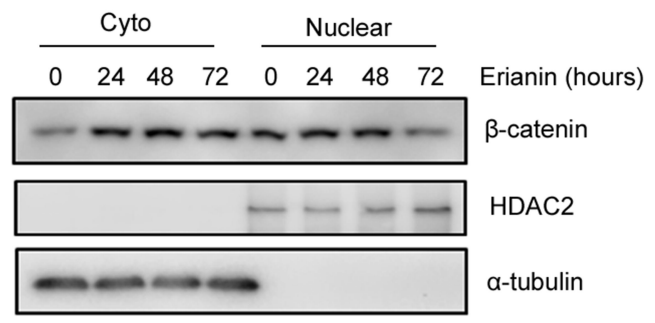

HCT116
B

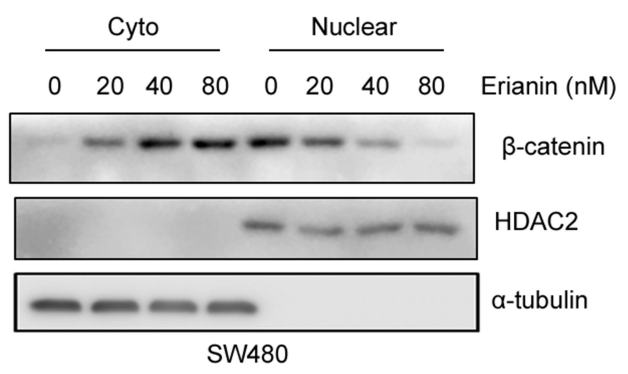

C

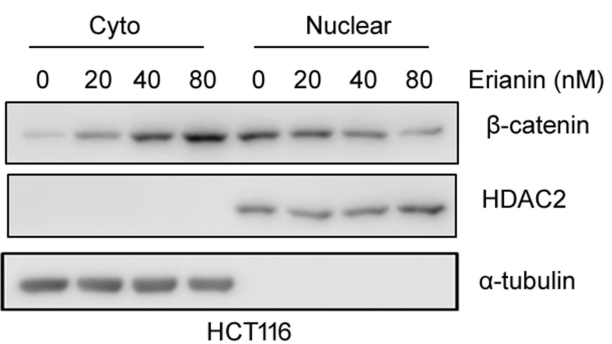

F

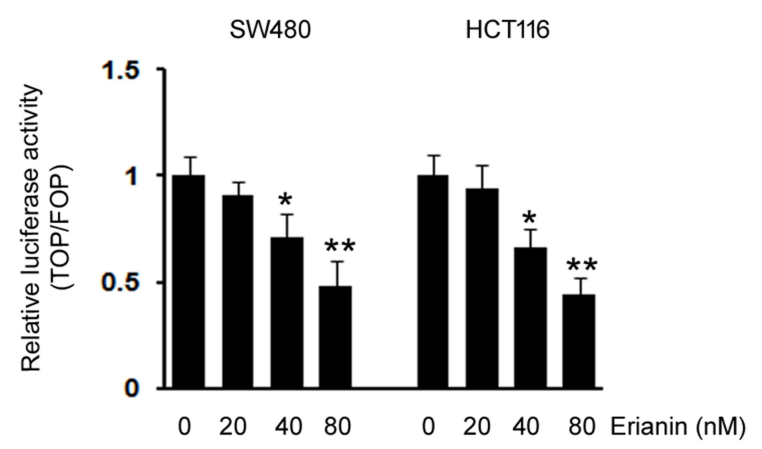

G

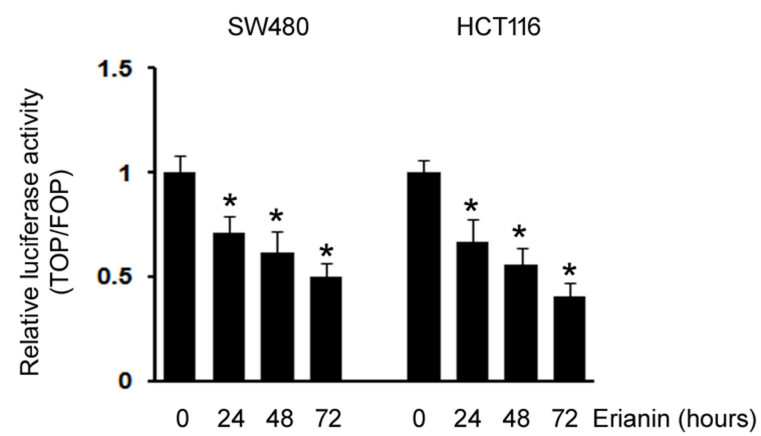

Figure 3 Erianin inhibited $\beta$-catenin translocation. (A) The protein level of indicated proteins was analyzed by Western blotting after being treated with indicated concentration of erianin for $24 \mathrm{~h}$. (B-E) The protein level of $\beta$-catenin in cytosol and nucleus was analyzed by Western blotting after treated with erianin for indicated concentration (B and $\mathbf{C}$ ) and time ( $\mathbf{D}$ and $\mathbf{E}$ ). ( $\mathbf{F}$ and $\mathbf{G})$ SW480 and HCTII6 cells were treated with erianin for indicated concentration (F) and time (G), the transcriptional activity of $\beta$-catenin was assessed by TOP/FOP luciferase reporter assay. $* P<0.05, * * P<0.0$ I.

\section{Erianin Inhibited the Expression of c-Myc} and Cyclin DI

As c-Myc and cyclin D1 are the direct targets of the Wnt/ $\beta$ catenin pathway, we then evaluated the mRNA and protein level of c-Myc and cyclin D1. Unsurprisingly, both mRNA and protein level of these two proteins were significantly decreased after erianin treatment (Figure 5A-C). Interestingly, no synergetic effect was observed when combining erianin with $\mathrm{Wnt} / \beta$-catenin signaling inhibitorWNT974, which indicated that erianin regulates c-Myc 

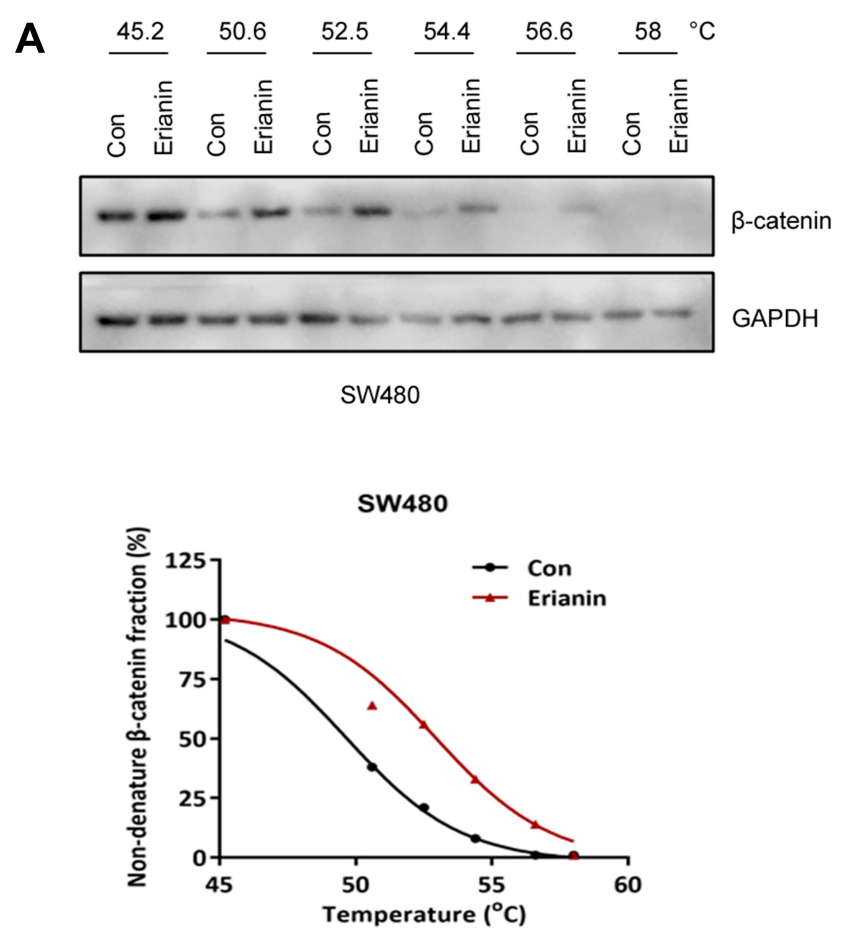

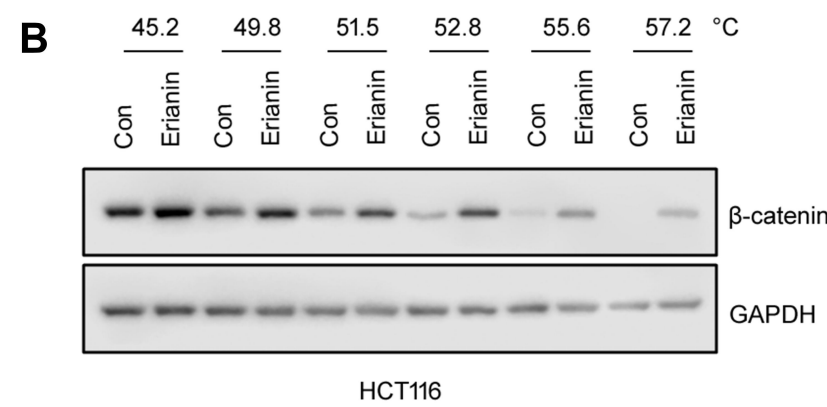

HCT116

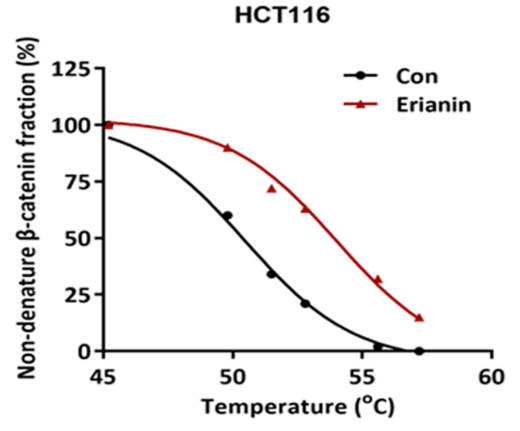

Figure 4 Erianin interacted with $\beta$-catenin. (A and B) SW480 (A) and HCTII6 (B) Cells were treated with $10 \mu$ M MGI32 for one hour followed by four hours incubation with $80 \mathrm{nM}$ erianin before performing thermal shift assay. The lower panel shows the charts of percentages of nondenatured protein fraction.

and cyclin D1 via Wnt/ $\beta$-catenin signaling (Figure $5 \mathrm{D}$ ). Furthermore, the inhibitory effect of erianin on c-Myc and cyclin D1 expression and cell viability could be reversed by $\beta$-catenin overexpression (Figure $5 \mathrm{E}$ and $\mathrm{F}$ ), which indicated that erianin regulates $\mathrm{CRC}$ cell growth via $\beta$-catenin.

\section{Erianin Decreased CD47 Expression and Increased Phagocytosis}

The immune checkpoint protein CD47 is included in the list of $\mathrm{Wnt} / \beta$-catenin target molecules with a role in immunity escape. ${ }^{17}$ Since $\beta$-catenin depletion by siRNA inhibited the expression of CD47 (Figure 6A), we then sought to know whether erianin regulates the expression of CD47. First, we explored the effects of erianin on CD47 mRNA, protein, and cell surface level. In both SW480 and HCT116 cells, erianin treatment significantly decreased the mRNA, protein, and cell surface level of CD47 (Figure 6B-D). Promoter analysis by UCSC genome browser demonstrates that H3K27 acetyl marks are enriched in CD47 promoter regions (Figure 6E). Next, our ChIP assay demonstrated that H3K27AC enrichment specifically near promoter region F3-F5 was significantly decreased with erianin treatment (Figure 6F). To investigate the effect of erianin on
CD47 mediated phagocytosis, we used an in vitro assay by co-culturing THP1 derived macrophage with CRC cell lines SW480 or HCT116. As shown in Figure 6G and H, treatment of erianin markedly promote colorectal cancer cell phagocytosis by macrophages. These results suggest that erianin treatment can attenuate CD47 expression and ultimately promote phagocytosis of CRC cells.

\section{Erianin Inhibited Tumor Growth In Vivo}

To investigate the possibility of erianin as a potential therapy in CRC, we tested the function of erianin on tumor growth in a mouse model. The mouse model was established by s. c. injection of SW480 cells into NOD/SCID mice. After three weeks treatment, we analyzed the tumor size and weight. As shown in Figure 7A-C, the tumor size and weight from the erianin treatment group were significantly lower than that from the control group. In addition, after 28 days of bearing tumor, the weight of the mice had no significant change (Figure 7D).

To examine the impact of therapy on $\beta$-catenin and its downstream signaling, localization of $\beta$-catenin, protein level of CD47, c-Myc, Bcl-2 and Bax, three representative tumors from each group were analyzed using Western blotting. As shown in Figure 7E and F, $\beta$-catenin expression in cytoplasm was increased; whereas expression in nucleus was decreased with the treatment of erianin. The 
A

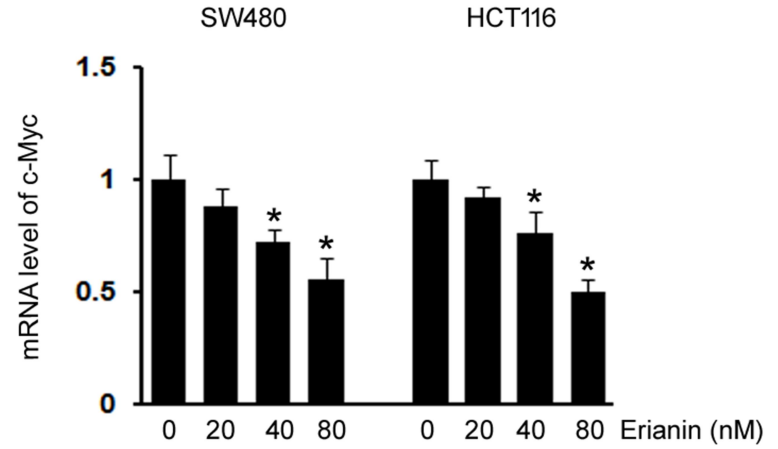

B

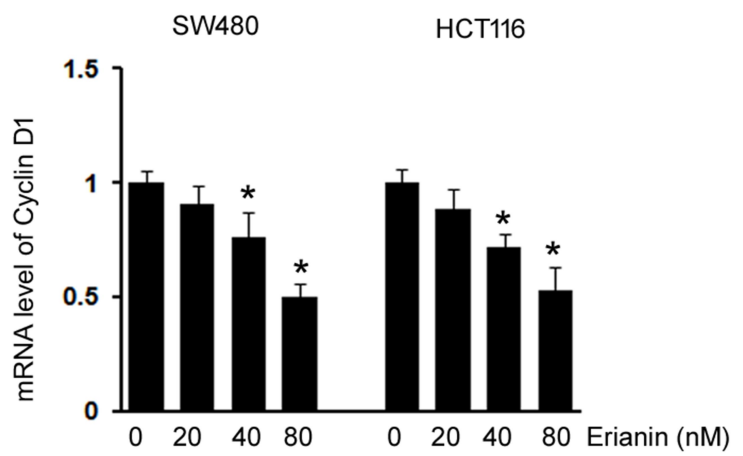

C SW480 HCT116
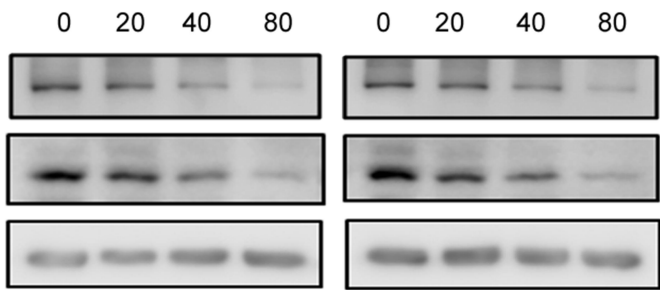

D

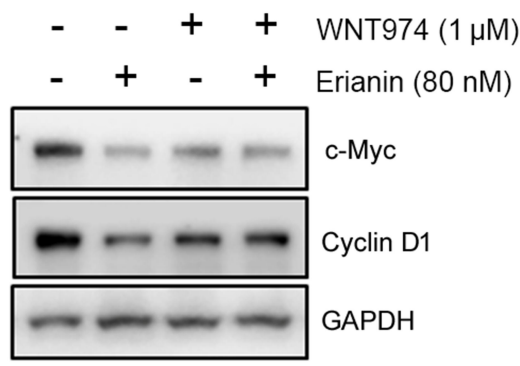

E

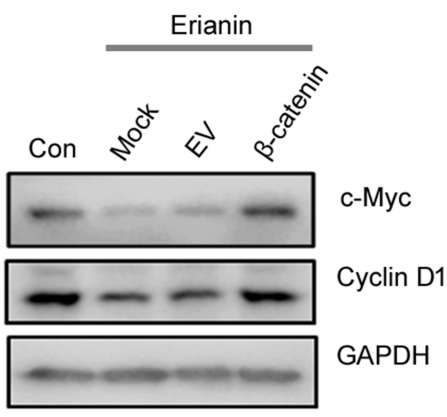

F

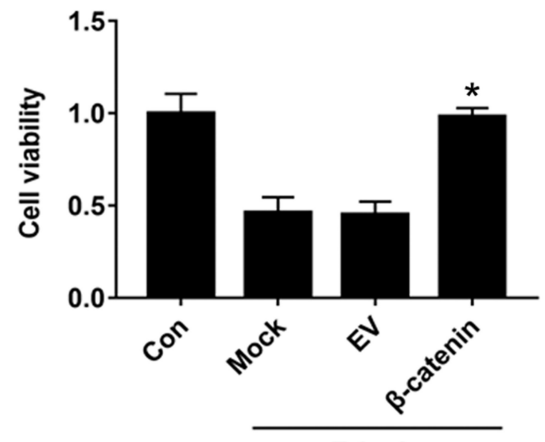

Erianin

Figure 5 Erianin inhibited the expression of c-Myc and cyclin DI. (A-C) After treated with indicated concentration and time of erianin, mRNA and protein level of c-Myc and cyclin DI were analyzed by qRT-PCR and Western blotting. $* P<0.05$. (D) SW480 cells were treated with erianin or/and WNT974 for $48 \mathrm{~h}$, protein levels of c-Myc and cyclin DI were analyzed by Western blotting. (E and F) SW480 cells were treated with erianin for $48 \mathrm{~h}$ followed by overexpression with $\beta$-catenin plasmid for $24 \mathrm{~h}$, protein levels of c-Myc and cyclin DI were analyzed by Western blotting $(\mathbf{E})$, and cell viability was assessed by CCK 8 assay $(\mathbf{F})$. $* P<0.05$.

protein level of CD47, c-Myc, and Bcl-2 decreased, while Bax increased after erianin treatment. These data indicated that erianin inhibited tumor growth via $\beta$-catenin in vivo.

\section{Discussion}

$\mathrm{CRC}$ is one of the most malignant and commonly diagnosed solid tumors all around the world. ${ }^{18-20}$ Although CRC incidence rates have declined somewhat, chemotherapies are inefficient in most CRC patients due to the development of acquired resistance..$^{21,22}$ Thus, researching novel and safe treatment strategies is essential for improving the prognosis of CRC patients. In recent years, natural medicinal plants are receiving more and more attention, and considered to be important sources of novel therapeutic drugs for cancer treatment. ${ }^{23}$ Dendrobium is considered as one of the most important herbs in the Orchidaceae family, and shows diverse pharmacological functions including anticancer, neuroprotective, antidiabetic, and immune-modulating activities. ${ }^{24}$ Erianin, derived from Dendrobium, is one of the most 

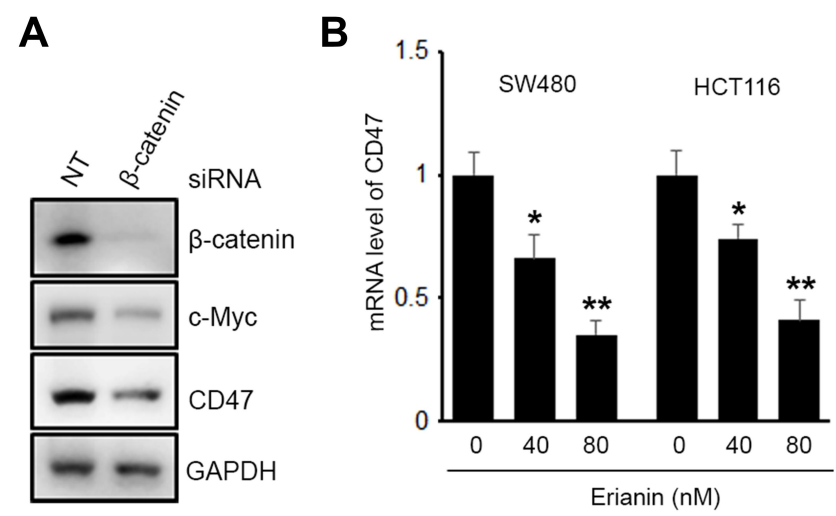

D

C
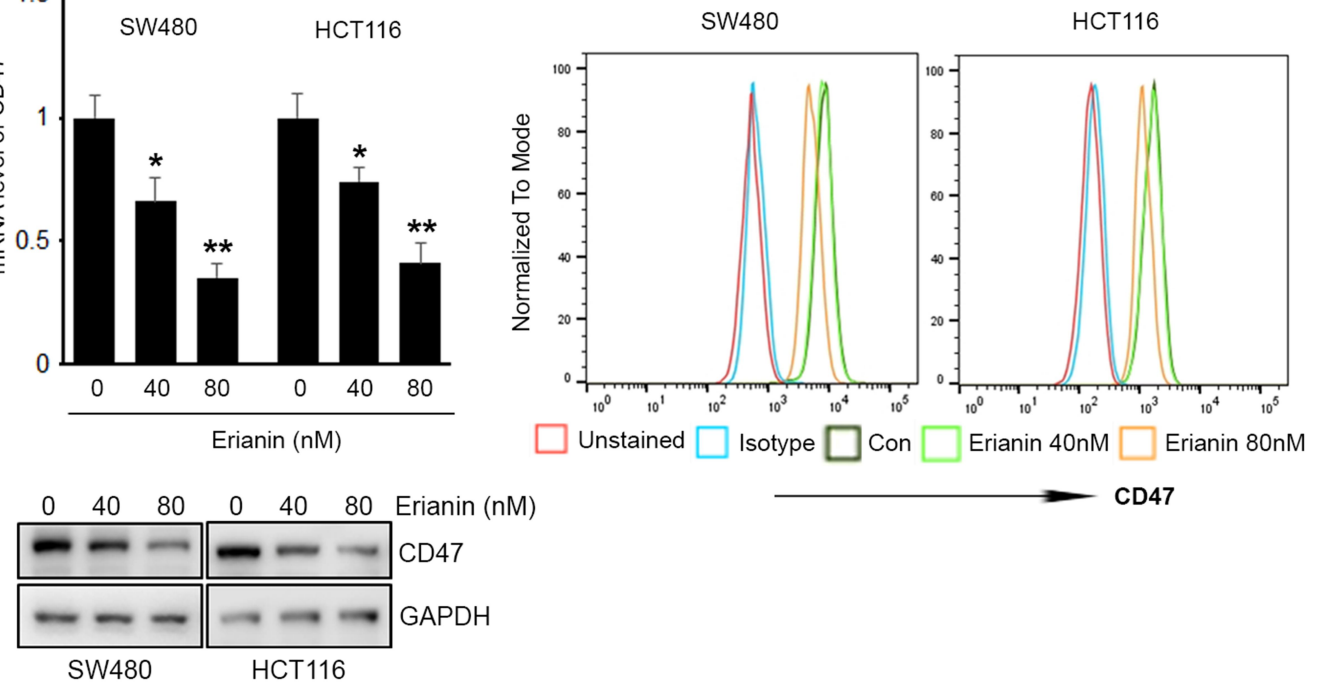

F
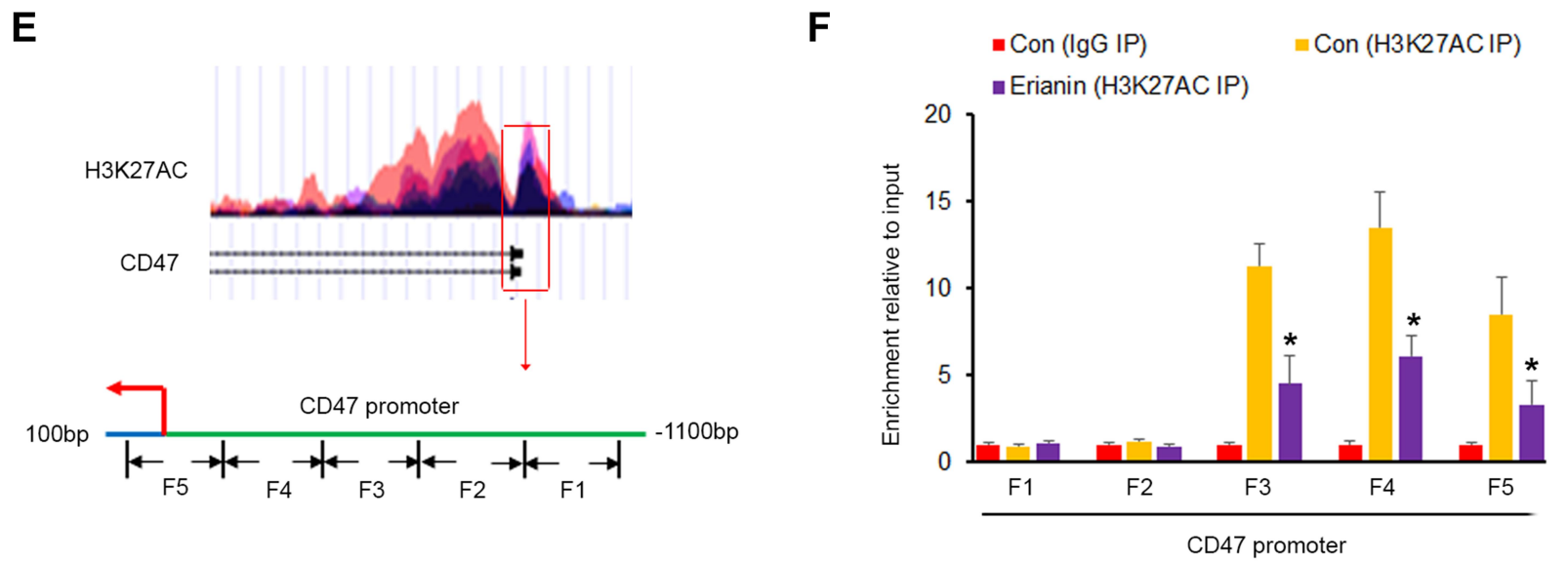

G

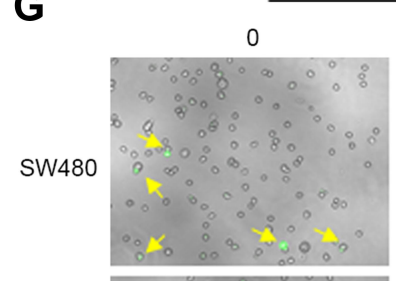

HCT116

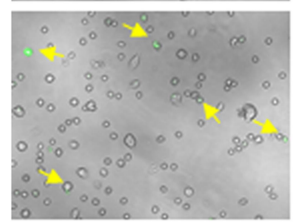

Erianin (nM)

40
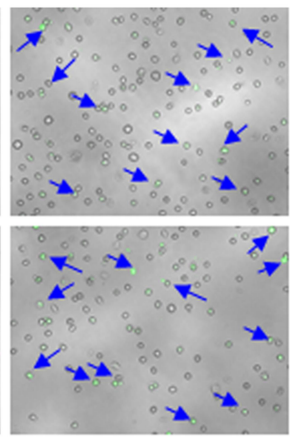

80

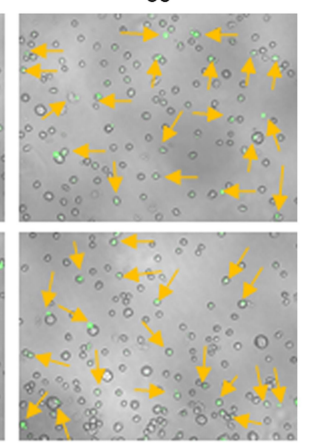

H

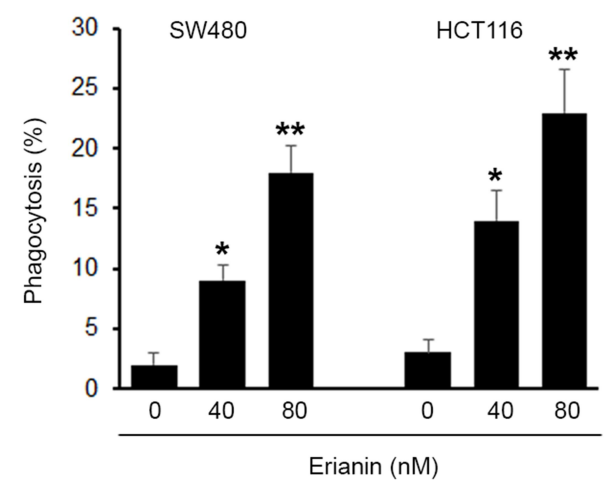

Figure 6 Erianin decreased CD47 expression and increased phagocytosis. (A) SW480 cells were transfected with nontarget (NT) or $\beta$-catenin siRNA for 72 h, protein levels of indicated protein weres measured by Western blotting. (B-D) SW480 and HCTII 6 cells were treated with erianin for indicated dose, the mRNA level (B), protein level $(\mathbf{C})$ and cell surface CD47 (D) were detected by qRT-PCR and flow. (E) The UCSC genome browser revealed the enrichment of H3K27AC on CD47 promoter. (F) The enrichment of H3K27AC on CD47 promoter (FI-F6) was detected by ChIP assay. (G and $\mathbf{H})$ SW480 and HCTI I6 were treated with indicated concentration of erianin for $48 \mathrm{~h}$, representative images showed the effect of erianin on phagocytosis $(\mathbf{G})$ and bar graphs showed quantitative analysis of phagocytosis $(\mathbf{H})$. $* P<0.05$, $* * P<0.01$. 
A

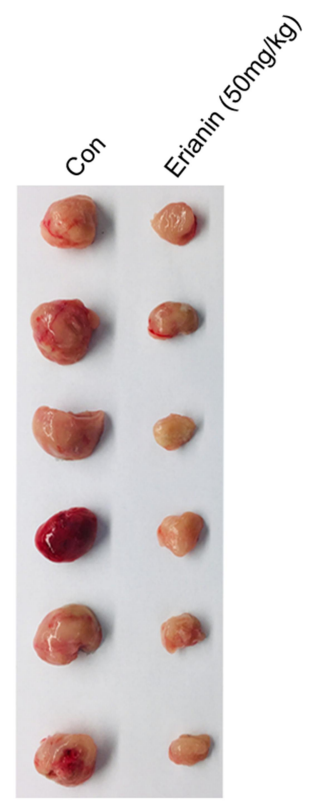

C

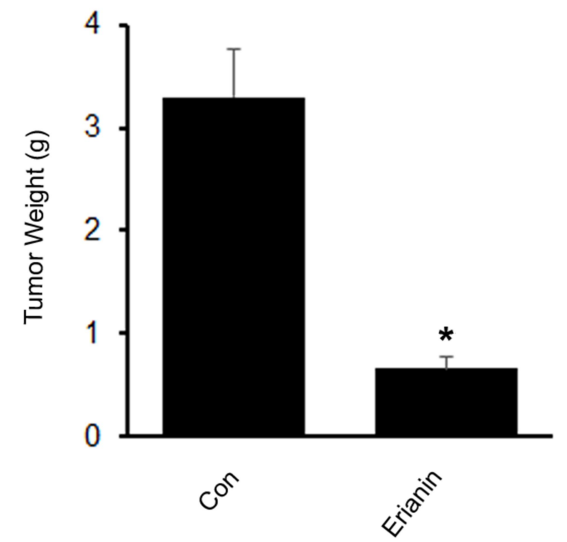

B

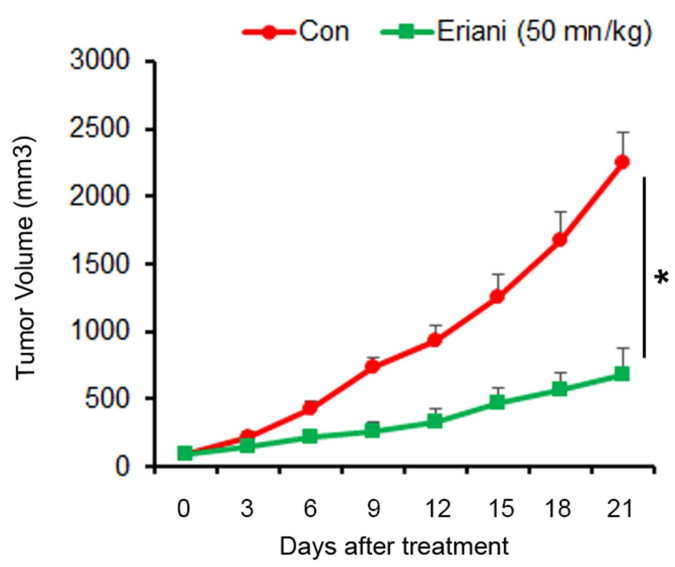

D

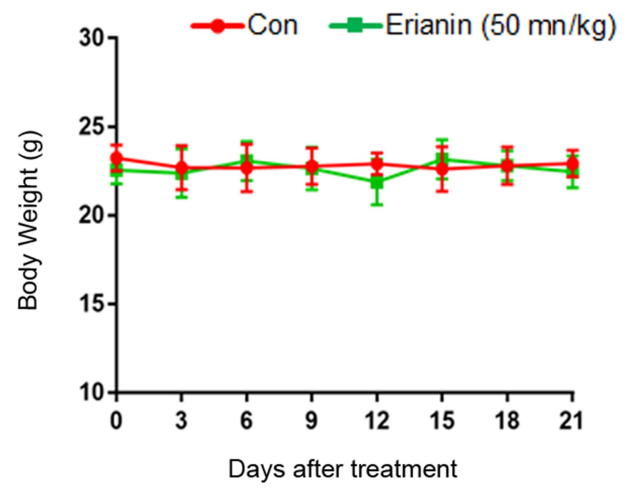

$\mathbf{F}$

Figure 7 Erianin inhibited tumor growth in vivo. (A) Typical photos of tumors from the control and erianin treated groups. (B and C) Erianin decreased tumor volume and weight. $* P<0.001$. (D) Mice body weight of control and erianin treated groups was measure at indicated time. (E) The protein level of $\beta$-catenin in cytosol and nucleus in three representative tumors from mouse \#I to mouse \# 3 of each group were analyzed by Western blotting. (F) The protein level of indicated protein in three representative tumors from mouse \#I to mouse \# 3 of each group were analyzed by Western blotting. 
noteworthy constituents that have been used as an antipyretic and an analgesic in traditional Chinese medicine. ${ }^{25}$ Recently, several studies have proved that erianin shows significant antitumour activity in a variety of human cancer cells. ${ }^{10-16}$ Consistent with literature, in this study, we found that Erianin had a significant antiproliferative effect against CRC cells. The inhibitory effect caused by erianin may result from induction of apoptosis and arrest of cell cycle at G2-M. Since the effect of erianin on CRC cells has never been studied before, we further confirm its antitumor activity in a mouse model, which indicated that erianin significantly inhibited tumor growth in vivo.

Several signaling pathways, including EGFR/MAPK, PI3K/AKT, or Wnt/ $\beta$-catenin have been linked to CRC genesis and progression. ${ }^{26}$ As the aberrant activation is present in almost all CRC cases, Wnt/ $\beta$-catenin signaling is prominent among these pathways. ${ }^{27}$ Inactivated mutations in the $A P C$ gene leads to stabilization and ensuing nuclear translocation of $\beta$-catenin to facilitate TCF/LEFdependent transcription of $\mathrm{Wnt} / \beta$-catenin signaling target genes, such as c-Myc and cyclin D1, to drive cell proliferation, survival, and metastasis. ${ }^{28-30}$ To understand the mechanisms of action of erianin, we assessed the effect of erianin on $\mathrm{Wnt} / \beta$-catenin pathway. Interestingly, we found that erianin treatment had no effect on $\beta$-catenin phosphorylation, but inhibited the translocation of $\beta$-catenin in the nucleus, which suggested to us that erianin physically interacts with $\beta$-catenin. Our cellular thermal shift assay confirmed this hypothesis, the thermal stability of $\beta$ catenin increased after erianin treatment. As $\beta$-catenin downstream targets, the expression level of c-Myc and cyclin D1 significantly decreased after erianin treatment.

CD47, a transmembrane glycoprotein, expresses ubiquitously and mediates a "self/do-not-eat-me" signal on normal cells. However, CD47 is often upregulated in tumor cells to evade innate immunity. ${ }^{31-33}$ Anti-CD47 antibodies, which block CD47- SIRP- $\alpha$ interactions and promote macrophagemediated phagocytosis of tumor cells, has shown promise in several solid tumors. ${ }^{31}$ In colorectal cancer, CD47 promotes colon cancer cell migration and metastasis. ${ }^{34}$ In addition, upregulated immune-escape pathways, such as CD47SIRP- $\alpha$, are responsible for immune escape and survival in circulating tumor cells of colorectal cancer. ${ }^{35} \mathrm{MYC}$, an oncogene identified as a Wnt/ $\beta$-catenin target gene, was reported to control CD47 transcription. Therefore, mutations in components of the $\mathrm{Wnt} / \beta$-catenin signaling pathway, which induced aberrant $M Y C$ expression, increased the expression of the CD47. In contrast, $M Y C$ inactivation led to a decrease in the expression of $\mathrm{CD} 47$, and caused the accumulation of tumor-associated macrophages. ${ }^{36}$ As erianin inhibited the transcriptional activity of $\beta$-catenin and c-Myc expression, we believed that erianin could also inhibit CD47 expression and phagocytosis. As shown in Figure 6, erianin inhibited C47 expression by decreasing the H3K27 acetyl marks enrichment in CD47 promoter regions. Consequently, macrophage- mediated phagocytosis improved.

\section{Conclusion}

Overall, erianin showed dual effects on cell growth and phagocytosis in human colorectal cells. This is the first study to investigate erianin's function in colorectal cells and we can infer erianin as a promising anticancer agent.

\section{Author Contributions}

All authors contributed towards data analysis, drafting and critically revising the paper, gave final approval of the version to be published, and agreed to be accountable for all aspects of the work.

\section{Disclosure}

The authors declare no conflicts of interest in this work.

\section{References}

1. Bermudez M, Aguilar-Medina M, Lizarraga-Verdugo E, et al. LncRNAs as regulators of autophagy and drug resistance in colorectal cancer. Front Oncol. 2019;9:1008. doi:10.3389/fonc.2019.01008

2. Fearon ER. Molecular genetics of colorectal cancer. Annu Rev Pathol. 2011;6:479-507. doi:10.1146/annurev-pathol-011110-130235

3. Strum WB. Colorectal Adenomas. N Engl J Med. 2016;375:389-390. doi:10.1056/NEJMc1604867

4. Thrumurthy SG, Thrumurthy SS, Gilbert CE, Ross P, Haji A. Colorectal adenocarcinoma: risks, prevention and diagnosis. BMJ. 2016;354:i3590. doi:10.1136/bmj.i3590

5. Nordlinger B, Sorbye H, Glimelius B, et al.;Group EG-ITC, Cancer Research UK, Arbeitsgruppe Lebermetastasen und-tumoren in der Chirurgischen Arbeitsgemeinschaft O, Australasian Gastro-Intestinal Trials G and Federation Francophone de Cancerologie D. Perioperative chemotherapy with FOLFOX4 and surgery versus surgery alone for resectable liver metastases from colorectal cancer (EORTC Intergroup trial 40983): a randomised controlled trial. Lancet. 2008;371:1007-1016. doi:10.1016/S0140-6736(08)60455-9

6. Longley DB, Harkin DP, Johnston PG. 5-fluorouracil: mechanisms of action and clinical strategies. Nat Rev Cancer. 2003;3:330-338. doi:10.1038/nrc1074

7. Meyerhardt JA, Mayer RJ. Systemic therapy for colorectal cancer. $N$ Engl J Med. 2005;352:476-487. doi:10.1056/NEJMra040958

8. Luk JM, Wang X, Liu P, et al. Traditional Chinese herbal medicines for treatment of liver fibrosis and cancer: from laboratory discovery to clinical evaluation. Liver Int. 2007;27:879-890. doi:10.1111/j.14783231.2007.01527.x

9. Teixeira da Silva JA, Ng TB. The medicinal and pharmaceutical importance of dendrobium species. Appl Microbiol Biotechnol. 2017;101:2227-2239. doi:10.1007/s00253-017-8169-9 
10. Li YM, Wang HY, Liu GQ. Erianin induces apoptosis in human leukemia HL-60 cells. Acta Pharmacol Sin. 2001;22:1018-1022.

11. Gong YQ, Fan Y, Wu DZ, Yang H, Hu ZB, Wang ZT. In vivo and in vitro evaluation of erianin, a novel anti-angiogenic agent. Eur $J$ Cancer. 2004;40:1554-1565. doi:10.1016/j.ejca.2004.01.041

12. Wang H, Zhang T, Sun W, et al. Erianin induces G2/M-phase arrest, apoptosis, and autophagy via the ROS/JNK signaling pathway in human osteosarcoma cells in vitro and in vivo. Cell Death Dis. 2016;7:e2247. doi:10.1038/cddis.2016.138

13. Zhu Q, Sheng Y, Li W, et al. Erianin, a novel dibenzyl compound in Dendrobium extract, inhibits bladder cancer cell growth via the mitochondrial apoptosis and JNK pathways. Toxicol Appl Pharmacol. 2019;371:41-54. doi:10.1016/j.taap.2019.03.027

14. Sun J, Fu X, Wang Y, et al. Erianin inhibits the proliferation of T47D cells by inhibiting cell cycles, inducing apoptosis and suppressing migration. Am J Transl Res. 2016;8:3077-3086.

15. Su C, Zhang P, Liu J, Cao Y. Erianin inhibits indoleamine 2, 3-dioxygenase -induced tumor angiogenesis. Biomed Pharmacother. 2017;88:521-528. doi:10.1016/j.biopha.2017.01.090

16. Zhang X, Wang Y, Li X, Yang A, Li Z, Wang D. The anti-carcinogenesis properties of erianin in the modulation of oxidative stress-mediated apoptosis and immune response in liver cancer. Aging (Albany NY). 2019;11:10284-10300. doi:10.18632/aging.102456

17. Gowda P, Patrick S, Singh A, Sheikh T, Sen E. Mutant isocitrate dehydrogenase 1 disrupts PKM2-beta-catenin-BRG1 transcriptional network-driven CD47 expression. Mol Cell Biol. 2018;38. doi:10.1128/MCB.00001-18

18. Chibaudel B, Tournigand C, Andre T, de Gramont A. Therapeutic strategy in unresectable metastatic colorectal cancer. Ther Adv Med Oncol. 2012;4:75-89. doi:10.1177/1758834011431592

19. Gustavsson B, Carlsson G, Machover D, et al. A review of the evolution of systemic chemotherapy in the management of colorectal cancer. Clin Colorectal Cancer. 2015;14:1-10. doi:10.1016/j.clcc.2014.11.002

20. Liu Z, Yu M, Fei B, Sun J, Wang D. Identification of natural compound derivative for inhibition of XLF and overcoming chemoresistance in colorectal cancer cells. Drug Des Devel Ther. 2019;13:3823-3834. doi:10.2147/DDDT.S215967

21. Zhang N, Yin Y, Xu SJ, Chen WS. 5-Fluorouracil: mechanisms of resistance and reversal strategies. Molecules. 2008;13:1551-1569. doi:10.3390/molecules 13081551

22. Temraz S, Mukherji D, Alameddine R, Shamseddine A. Methods of overcoming treatment resistance in colorectal cancer. Crit Rev Oncol Hematol. 2014;89:217-230. doi:10.1016/j.critrevonc.2013.08.015

23. Kudumela RG, McGaw LJ, Masoko P. Antibacterial interactions, anti-inflammatory and cytotoxic effects of four medicinal plant species. BMC Complement Altern Med. 2018;18:199. doi:10.1186/ s12906-018-2264-z
24. Baek JM, Kim JY, Ahn SJ, et al. Dendrobium moniliforme exerts inhibitory effects on both receptor activator of nuclear factor kappa-B ligand-mediated osteoclast differentiation in vitro and lipopolysaccharide-induced bone erosion in vivo. Molecules. 2016;21:295. doi:10.3390/molecules21030295

25. Ng TB, Liu F, Wang ZT. Antioxidative activity of natural products from plants. Life Sci. 2000;66:709-723. doi:10.1016/S0024-3205(99) 00642-6

26. Koveitypour Z, Panahi F, Vakilian M, et al. Signaling pathways involved in colorectal cancer progression. Cell Biosci. 2019;9:97. doi:10.1186/s13578-019-0361-4

27. Schatoff EM, Leach BI, Dow LE. Wnt signaling and colorectal cancer. Curr Colorectal Cancer Rep. 2017;13:101-110. doi:10. 1007/s11888-017-0354-9

28. Basu S, Haase G, Ben-Ze'ev A. Wnt signaling in cancer stem cells and colon cancer metastasis. F1000Res. 2016;5:699. doi:10.12688/ f1000research.7579.1

29. Cheng X, Xu X, Chen D, Zhao F, Wang W. Therapeutic potential of targeting the Wnt/beta-catenin signaling pathway in colorectal cancer. Biomed Pharmacother. 2019;110:473-481. doi:10.1016/j.biopha. 2018.11.082

30. Li Q, Lai Q, He C, et al. RUNX1 promotes tumour metastasis by activating the Wnt/beta-catenin signalling pathway and EMT in colorectal cancer. J Exp Clin Cancer Res. 2019;38:334. doi:10.1186/ s13046-019-1330-9

31. Willingham SB, Volkmer JP, Gentles AJ, et al. The CD47-signal regulatory protein alpha (SIRPa) interaction is a therapeutic target for human solid tumors. Proc Natl Acad Sci $U$ S A. 2012;109:6662-6667. doi:10.1073/pnas.1121623109

32. Tseng D, Volkmer JP, Willingham SB, et al. Anti-CD47 antibody-mediated phagocytosis of cancer by macrophages primes an effective antitumor T-cell response. Proc Natl Acad Sci U S A. 2013;110:11103-11108. doi:10.1073/pnas.1305569110

33. Chen J, Zhong MC, Guo H, et al. SLAMF7 is critical for phagocytosis of haematopoietic tumour cells via Mac-1 integrin. Nature. 2017;544:493-497. doi:10.1038/nature22076

34. Zhang Y, Sime W, Juhas M, Sjolander A. Crosstalk between colon cancer cells and macrophages via inflammatory mediators and CD47 promotes tumour cell migration. Eur J Cancer. 2013;49:3320-3334. doi:10.1016/j.ejca.2013.06.005

35. Steinert G, Scholch S, Niemietz T, et al. Immune escape and survival mechanisms in circulating tumor cells of colorectal cancer. Cancer Res. 2014;74:1694-1704. doi:10.1158/0008-5472.CAN-13-1885

36. Casey SC, Tong L, Li Y, et al. MYC regulates the antitumor immune response through CD47 and PD-L1. Science. 2016;352:227-231. doi:10.1126/science.aac9935

\section{Publish your work in this journal}

Drug Design, Development and Therapy is an international, peerreviewed open-access journal that spans the spectrum of drug design and development through to clinical applications. Clinical outcomes, patient safety, and programs for the development and effective, safe, and sustained use of medicines are a feature of the journal, which has also been accepted for indexing on PubMed Central. The manuscript management system is completely online and includes a very quick and fair peer-review system, which is all easy to use. Visit http://www. dovepress.com/testimonials.php to read real quotes from published authors. 